\title{
Coherent Many-Body Spin Dynamics in a Long-Range Interacting Ising Chain
}

\author{
Johannes Zeiher, ${ }^{1,}$ Jae-yoon Choi, ${ }^{1}$ Antonio Rubio-Abadal, ${ }^{1}$ Thomas Pohl, ${ }^{2}$ \\ Rick van Bijnen, ${ }^{3}$ Immanuel Bloch, ${ }^{1,4}$ and Christian Gross ${ }^{1}$ \\ ${ }^{1}$ Max-Planck-Institut für Quantenoptik, 85748 Garching, Germany \\ ${ }^{2}$ Department of Physics and Astronomy, Aarhus University, DK 8000 Aarhus C, Denmark \\ ${ }^{3}$ Institut für Quantenoptik und Quanteninformation, \\ Österreichische Akademie der Wissenschaften, 6020 Innsbruck, Austria \\ ${ }^{4}$ Fakultät für Physik, Ludwig-Maximilians-Universität München, 80799 München, Germany \\ (Received 28 August 2017; revised manuscript received 16 October 2017; published 14 December 2017) \\ Coherent many-body quantum dynamics lies at the heart of quantum simulation and quantum computation. \\ Both require coherent evolution in the exponentially large Hilbert space of an interacting many-body system. To \\ date, trapped ions have defined the state of the art in terms of achievable coherence times in interacting spin \\ chains. Here, we establish an alternative platform by reporting on the observation of coherent, fully interaction- \\ driven quantum revivals of the magnetization in Rydberg-dressed Ising spin chains of atoms trapped in an \\ optical lattice. We identify partial many-body revivals at up to about ten times the characteristic time scale set by \\ the interactions. At the same time, single-site-resolved correlation measurements link the magnetization \\ dynamics with interspin correlations appearing at different distances during the evolution. These results mark \\ an enabling step towards the implementation of Rydberg-atom-based quantum annealers, quantum simulations \\ of higher-dimensional complex magnetic Hamiltonians, and itinerant long-range interacting quantum matter. \\ DOI: 10.1103/PhysRevX.7.041063 \\ Subject Areas: Atomic and Molecular Physics, \\ Condensed Matter Physics
}

\section{INTRODUCTION}

The coherent unitary evolution of closed many-body quantum systems initially prepared in a superposition of different eigenstates is one of the most fundamental concepts of quantum theory. It predicts a fast dephasing of the initial state, followed by its revival after long times. This dynamics originates from the discrete energy spectrum of the many-body eigenstates, each evolving with its characteristic frequency. The expected collapse and revival dynamics is in stark contrast to the experience that in typical interacting macroscopic systems, quantum revivals are entirely absent. This is a consequence of the exponential increase of the number of distinct energy levels with system size, making the spectrum effectively continuous, such that the revival time diverges. Even in small systems with few constituents, the observation of quantum revivals is far from trivial because of residual couplings to the (macroscopic) environment causing decoherence. This renders the observation of quantum revivals in a many-body system one of the most demanding tests to demonstrate its coherent evolution, which itself is indispensable, for example, for efficient adiabatic quantum computation $[1,2]$.

*johannes.zeiher@mpq.mpg.de

Published by the American Physical Society under the terms of the Creative Commons Attribution 4.0 International license. Further distribution of this work must maintain attribution to the author(s) and the published article's title, journal citation, and DOI.
Quantum revivals have so far been observed experimentally using appropriate observables in few-particle systems, whose dynamics was additionally constrained to small parts of the Hilbert space. Seminal achievements are the observation of collapse and revivals of Rabi oscillation dynamics of Rydberg states coupled to the radiation field in a microwave cavity $[3,4]$, of revivals of the motional state of a single trapped ion [5], or of coherent photonic states in microwave resonators coupled to a transmon qubit [6]. Revival dynamics has also been observed for small coherent states of matter realized with ultracold atoms in individual sites of optical lattices. The on-site interactions between the indistinguishable atoms led first to dephasing and later to revivals of the relative phase of the condensate wave function on the different lattice sites $[7,8]$.

A much more complex many-body dynamics is expected when the full Hilbert space is accessible in the time evolution, and in particular in systems with long-range interactions. Quantum magnets featuring such interactions have been realized recently with trapped ions [9-12], ground state molecules [13], magnetic atoms [14], and neutral atoms coupled to Rydberg states on resonance $[15,16]$ or off resonantly $[17,18]$. Indeed, indications for partial quantum revivals have been detected in the dynamics of the next-to-nearest-neighbor correlations [11] and in the magnetization of small systems of three spins [19].

Here, we take advantage of the revival dynamics expected solely in closed quantum systems to benchmark the coherence of interaction-induced spin dynamics in 
(a)

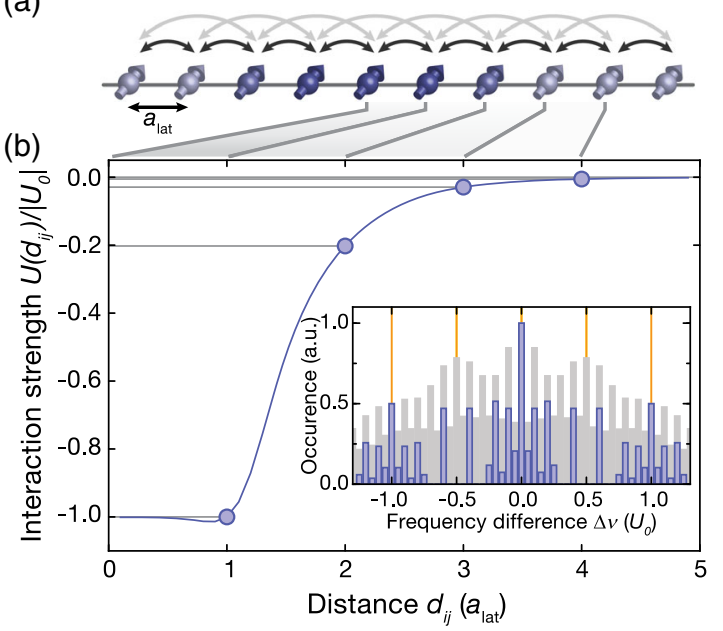

(c) Prob. $|\rightarrow\rangle,|\leftarrow\rangle \quad$ Spin right, $|\rightarrow\rangle \quad$ Spin left, $|\leftarrow\rangle$

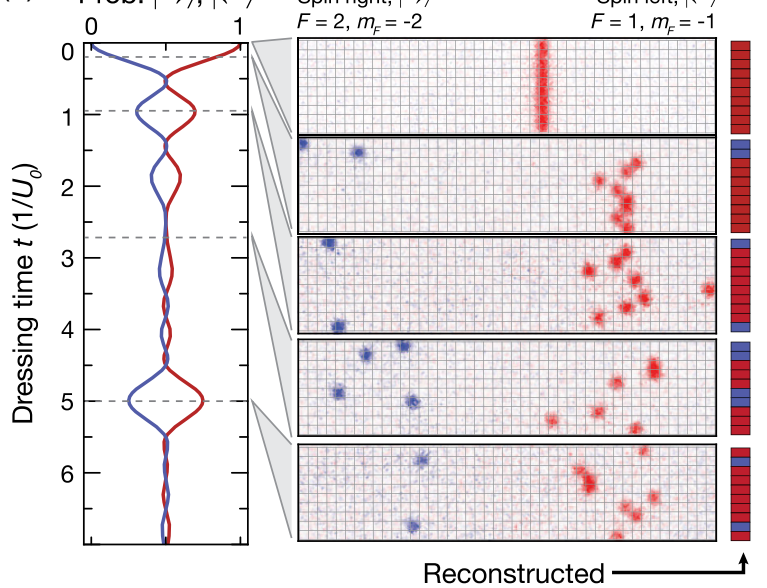

FIG. 1. Schematic of the Ising spin chain and spin-selective detection. (a) Illustration of a spin chain with $N=10$ spins initialized in the fully transverse magnetized state. The dominant contributions of the Rydberg-dressed interaction between nearest and next-tonearest spins spaced by $a_{\text {lat }}$ are indicated by the dark gray and light gray arrows. The brightness of the blue color of the spins encodes the interaction strength between the exemplary selected fifth spin with the rest of the chain. The gray lines are guides to the eye to link to the interaction potential shown in (b). (b) Ab initio calculated Rydberg-dressed potential [18] for our experimental parameters $\Omega / 2 \pi=$ $3.57(3) \mathrm{MHz}$ and $\Delta / 2 \pi=11.00(2) \mathrm{MHz}$, normalized to the nearest-neighbor interaction strength $\left|U_{0}\right|=13.1(5) \mathrm{kHz}$ (blue solid line), with the relevant potential at multiples of the lattice distance $a_{\text {lat }}$ marked by blue points and gray horizontal lines. The inset shows the occurrence of frequency differences $\Delta \nu$ in the many-body spectrum of the long-range interacting Ising model (gray bars) and those governing transverse magnetization dynamics (blue bars). Orange vertical lines mark the corresponding $\Delta \nu$ for the Ising model with nearest-neighbor interactions only. (c) Simulated revival dynamics of the populations of spin left (red) and spin right (blue), starting from the initially fully magnetized chain in the $S^{y}$ direction for a defect-free chain of ten spins and long-range interactions. Clear partial revivals are observed during the evolution. The fluorescence images to the right show characteristic spin configurations for ten spins observed during the collapse and revival dynamics at times indicated by the gray lines. The spin of the atoms was detected via an in situ Stern-Gerlach sequence, which led to a spatial separation of spin-left (red) and spin-right atoms (blue). This allows for the reconstruction of the full spin and density distribution (pictograms to the right).

Rydberg-dressed Ising chains of about ten atoms. We observe partial quantum revivals of the transverse magnetization of the chains up to times exceeding ten times the characteristic time scale set by the nearest-neighbor coupling strength. Single-spin resolved correlation measurements reveal the microscopic origin of the interactioninduced collapse dynamics at short times.

\section{REVIVAL DYNAMICS IN SPIN CHAINS}

In our experiments, we implemented a one-dimensional (1d) Ising spin chain of $N$ spins with soft-core-type long-range interactions using ultracold ${ }^{87} \mathrm{Rb}$ atoms in an optical lattice, optically "dressed" to a strongly interacting Rydberg state [20-22]; see Fig. 1(a). The spin-1/2 degree of freedom is encoded in two hyperfine ground states and its dynamics is described by the Hamiltonian

$$
\hat{H}=h \sum_{i \neq j}^{N} \frac{U\left(d_{i j}\right)}{2} \hat{S}_{i}^{z} \hat{S}_{j}^{z}
$$

Here, $\hat{S}_{i}^{z}$ denotes the spin operator measuring the spin in the $z$ direction at a lattice site $i$, and we omit all terms linear in the spin operators because they are irrelevant to the subsequent discussion [23]. For the chosen parameters of the optical coupling, the interaction potential $U\left(d_{i j}\right)$ is approximated by a soft-core shape for spins at a distance $d_{i j}=|i-j|$ [see Fig. 1(b)]. For distances smaller than the lattice spacing $d_{i j} \leq a_{\text {lat }}=532 \mathrm{~nm}$, it saturates to the nearest-neighbor value $U(1)=U_{0} \approx-13 \mathrm{kHz}$, and for larger distances, it asymptotically falls off with a van der Waals tail, $U\left(d_{i j}\right) \propto 1 / d_{i j}^{6}$ [18].

To study the quantum evolution, the system is initially prepared in a separable coherent spin state [24] with maximal magnetization along the $S^{y}$-direction, $\left|\psi_{0}\right\rangle=$ $(|\leftarrow\rangle)^{\otimes N}$, where $|\leftarrow\rangle$ is a single-spin eigenstate of $\hat{S}^{y}$. In the $\hat{S}^{z}$ basis, in which all many-body eigenstates of $\hat{H}$ factorize, each spin is equally likely found in the two single-spin eigenstates of $\hat{S}^{z}$. Thus, each many-body eigenstate $|\lambda\rangle$ with possibly degenerate eigenenergy $E_{\lambda}=$ $h \nu_{\lambda}$ is populated with equal probability $\left|\left\langle\lambda \mid \psi_{0}\right\rangle\right|^{2}=\left|c_{\lambda}\right|^{2}$ and an amplitude of $c_{\lambda}=2^{-N / 2} e^{i \phi_{\lambda}}$. After unitary evolution of $\left|\psi_{0}\right\rangle$ with $\hat{U}=e^{-i \hat{H} t / \hbar}$, the expectation value of the local transverse magnetization at site $j$ in the chain becomes $\left\langle\hat{S}_{j}^{y}(t)\right\rangle=2^{-N} \sum_{\lambda, \eta} e^{-i 2 \pi\left(\nu_{\lambda}-\nu_{\eta}\right) t}\left\langle\eta\left|\hat{S}_{j}^{y}\right| \lambda\right\rangle$, where the phases $\phi_{\lambda}$ and $\phi_{\eta}$ have been absorbed in the operator matrix element. The dynamics of $\left\langle\hat{S}_{j}^{y}(t)\right\rangle$ is hence determined by those frequency differences $\Delta \nu=\nu_{\lambda}-\nu_{\eta}$, for which 
the operator matrix element $\left\langle\eta\left|\hat{S}_{j}^{y}\right| \lambda\right\rangle$ does not vanish [see Fig. 1(b)]. Due to the contribution of different $\Delta \nu$ 's, the initial state and hence the magnetization is expected to undergo an initial collapse dynamics, followed by a revival on longer time scales. Importantly, this dynamics is purely driven by the buildup of correlations [12], since the initial state is a mean-field steady state with $\left\langle\hat{S}_{j}^{z}\right\rangle=0$. Indeed, the collapse is accompanied by the generation of entanglement at short evolution times in the form of spin squeezing [25-27]. At a later time $t=1 / 2 U_{0}$, the system realizes approximately a cluster state [28], a highly entangled state useful for quantum computation.

To obtain an intuitive understanding of the collapse and revival dynamics, it is illustrative to consider the case of an Ising model with only nearest-neighbor interactions of strength $h U_{0}$. For such a case, the transverse magnetization $\left\langle\hat{S}_{\text {tot }}^{y}\right\rangle=\sum_{j=1}^{N}\left\langle\hat{S}_{j}^{y}\right\rangle$ would show periodic revivals at times $t_{n}=n / U_{0}$. This is due to the highly degenerate spectrum with all relevant energy differences $\Delta \nu$ either vanishing, or being equal to $U_{0}$ for a spin located in the bulk or to $U_{0} / 2$ for a spin at the edge of the system [see Fig. 1(b) and [23]]. Adding interactions with a longer range leads to a more complex spectrum, breaking many of the degeneracies present in the former case. For the interaction potential realized in the experiment and a chain of $N=10$ atoms, the number of relevant frequency differences also increases significantly [see Fig. 1(b)]. Whereas this shifts a possible perfect revival of the initial state to experimentally inaccessible times, the magnetization may still show dynamics with partial revivals [see Fig. 1(c)]. The exact magnetization dynamics can be calculated analytically for our initial state, and it predicts that the local transverse magnetization evolves in time as $\left\langle\hat{S}_{j}^{y}(t)\right\rangle=\frac{1}{2} \prod_{i \neq j}^{N} \cos \left[\pi U\left(d_{i j}\right) t\right][25,29]$. This confirms the intuition that partial revivals of the magnetization of a single spin originate from its interaction $U\left(d_{i j}\right)$ with spins at different distances $d_{i j}$ and the resulting beat notes. Despite the conceptual similarities to previously studied revival dynamics [4-8], an important difference of the spin system with long-range interactions studied here is the absence of a spatial spin exchange symmetry, and hence, there is no simplifying description of the system's temporal dynamics in terms of symmetric Dicke states [30]. Thus, here the dynamics is in a regime exploring a much larger portion of the Hilbert space, leading also to an interesting spatial structure in the time evolution that we reveal by our microscopic detection [see Fig. 1(c) and [23]].

\section{MAGNETIZATION AND CORRELATION DYNAMICS}

Our experiments started with the preparation of an atomic chain of ten sites with 87(3)\% filling from a Mott insulator using single-site addressing techniques $[23,31,32]$. Subsequently, the coherent spin state was

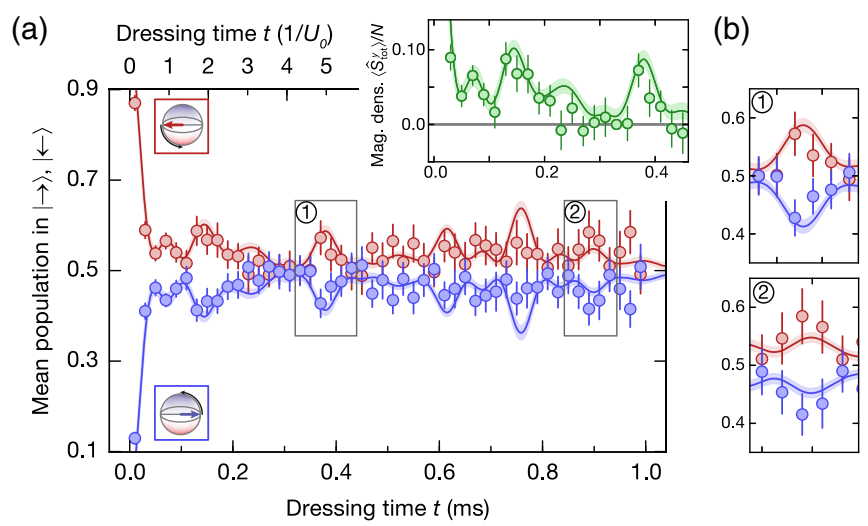

FIG. 2. Evolution of the mean magnetization density. (a) The probability to measure atoms in the states $|\leftarrow\rangle(|\rightarrow\rangle)$ vs total dressing time $t$ shown as red (blue) data points. The final spin rotation before detection is indicated by the pictograms (upper and lower left corner). The upper axis is scaled in units of the inverse nearest-neighbor interaction $1 /\left|U_{0}\right|=76(3) \mu$ s. The total atom number was restricted to be $N<15$ to filter out events with clear preparation errors. The solid line shows the theoretically expected dynamics, averaged over 100 initial chains randomly selected from a reference data set with an initial filling of $87(3) \%$ and a mean atom number of 10(1.4). The shaded region marks the corresponding standard error of the mean (s.e.m.). All data points are an average over at least 50 experimental realizations (150 for $t<0.1 \mathrm{~ms}$ ). The inset shows the initial dynamics of the mean transverse magnetization density $\left\langle\hat{S}_{\text {tot }}^{y}\right\rangle / N$ up to $t \approx 6 / U_{0}$, obtained from the spin populations (green data points) with the theoretical prediction (green solid line). (b) Zoom-in on two magnetization features at $t \approx 5 / U_{0}$ (1) and $t \approx 12 / U_{0}$ (2), as indicated by the gray boxes in (a). Even at long times up to $12 / U_{0}$, the nonvanishing magnetization indicates the presence of finite coherence. All error bars on the data points denote one s.e.m.

initialized by a global microwave-induced $\pi / 2$ rotation about the $S^{x}$-axis ending in an equal superposition of the hyperfine states $\left|F, m_{F}\right\rangle=|1,-1\rangle$ and $|2,-2\rangle$. Next, we switched on the interactions $U\left(d_{i j}\right)$ for a total time $t$ by illuminating the sample with the "dressing" laser, coupling to the $31 P_{1 / 2}$ Rydberg state with Rabi frequency $\Omega / 2 \pi=$ 3.57(3) $\mathrm{MHz}$ and detuning $\Delta / 2 \pi=11.00$ (2) MHz. This Rydberg dressing was interrupted after a time $t / 2$ to implement a spin-echo pulse of area $\pi$ about the $S^{x}$-axis to remove trivial phases accumulated because of single atom shifts proportional to $\hat{S}^{z}$, thereby leaving the spin-spin interaction as the only drive of the dynamics [23]. Finally, the spin along the $S^{y}$ direction was read out using a second $\pi / 2$ pulse to rotate the spins to the $\hat{S}^{z}$ basis, where we separated the spins in situ, making use of their different magnetic moments. This enabled a position-dependent readout of the spin direction [33] and provided access to the local and global magnetization, as well as the total atom number $N$ after the dynamics.

The collapse and revival dynamics of the initial coherent spin state is observed by tracking the evolution of the spin populations along the $S^{y}$ direction. This is equivalent 

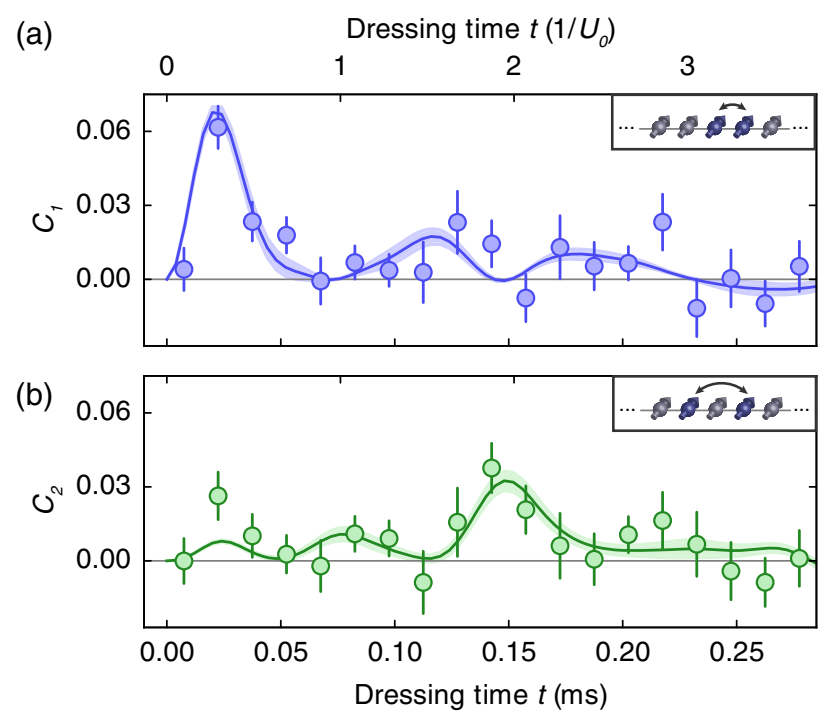

FIG. 3. Local characterization of the spin dynamics. (a) Observed evolution of the connected correlations $C_{d}$ for spins separated by $d=a_{\text {lat }}$ and (b) $d=2 a_{\text {lat }}$, as indicated by the pictograms. The solid line with surrounding shading shows the corresponding prediction of the same theoretical calculation as used in Fig. 2. All error bars denote one s.e.m.

to tracking the mean transverse magnetization density $\left\langle\hat{S}_{\text {tot }}^{y}(t)\right\rangle / N$, where the measured total atom number at time $t$ is used for the normalization (see Fig. 2). For short times, we find a fast interaction-induced depolarization dynamics up to a time of approximately $t \approx 40 \mu \mathrm{s} \approx 1 / 2 U_{0}$, before exhibiting clear partial revivals at $t \approx 1 / U_{0}, 2 / U_{0}$, and $5 / U_{0}$. The times of the revivals can be qualitatively explained by considering only the nearest and next-tonearest neighbor interaction, which differ by a factor of about 5 in our case. Interestingly, even at longer times, the data closely follow the numerical prediction, showing a finite magnetization signal up to $t=12 / U_{0}$ and approaching the $1 / e$ lifetime $\tau=1.21(3) \mathrm{ms}$ of the atom number in the chains. This indicates that the atom loss does not induce excessive dephasing, which would result in a vanishing magnetization density in the system. Our numerical simulation of the dynamics considers pure unitary evolution and additionally takes into account the randomness due to the initially imperfect filling of the chain. The presence of defects in the chain suppresses all revivals at odd multiples of $1 / U_{0}$ as an empty site causes the neighboring spin to evolve with approximately half the frequency [23].

Investigating spatial spin-spin correlations provides microscopic insight into the collapse and revival dynamics. To this end, we evaluated the connected spin-spin correlator $C_{d}=\left\langle\hat{S}_{i}^{y} \hat{S}_{i+d}^{y}\right\rangle-\left\langle\hat{S}_{i}^{y}\right\rangle\left\langle\hat{S}_{i+d}^{y}\right\rangle$ at different distances $d$ vs time (see Fig. 3). The correlation signal for neighboring spins $\left(C_{1}\right)$ reveals that the initial decay of the magnetization is driven by the buildup of strong nearest-neighbor correlations, peaking at $t \approx 1 / 4 U_{0}$. Whereas for pure nearest-neighbor interactions one solely expects correlations extending to the neighboring spins and $C_{2}=0$ for all times, we also find a nonzero next-to-nearest-neighbor correlator $C_{2}$, peaking later at $t \approx 2 / U_{0}$. In our case, a nonzero $C_{2}$ is therefore a direct manifestation of the long-range interactions. These can entangle distant parts of the system even in the absence of moving quasiparticles [10,11,34], which arise for example in the Ising model with transverse field as free fermions after a Jordan-Wigner transformation [30]. An illustrative interpretation of the initial strong growth of the nearest-neighbor correlation can be obtained by rewriting $\hat{H}_{\text {int }}$ in terms of the raising and lowering operators with respect to the $S^{y}$-direction, $\hat{\tilde{S}}_{j}^{ \pm}=\hat{S}_{j}^{x} \pm i \hat{S}_{j}^{z}$, yielding $\hat{H}=h \sum_{i \neq j}^{N}\left[U\left(d_{i j}\right) / 4\right]\left(\hat{\tilde{S}}_{i}^{+} \hat{\tilde{S}}_{j}^{-}+\hat{\tilde{S}}_{i}^{-} \hat{\tilde{S}}_{j}^{+}-\hat{\tilde{S}}_{i}^{+} \hat{\tilde{S}}_{j}^{+}-\hat{\tilde{S}}_{i}^{-} \hat{\tilde{S}}_{j}^{-}\right)$. Starting with $\left|\psi_{0}\right\rangle$, where all spins are in state $|\leftarrow\rangle$, only the last term in the sum contributes for short times, which flips spins pairwise. This "pair production" of $|\rightarrow\rangle$ spins is strongest at short distances, explaining the observed correlation signal qualitatively.

\section{PARITY DECAY AND DECOHERENCE}

All terms in the Hamiltonian above are parity conserving since the number of spins flipped from $|\leftarrow\rangle$ to $|\rightarrow\rangle$ can only be changed in steps of two. Hence, ideally, we expect to observe only even numbers of $|\rightarrow\rangle$ spins to appear during the evolution. This situation is similar to spontaneous pair creation in the squeezed vacuum state of photons. There, a strongly parity-modulated signal in the excitation numbers [35] has also been observed and interpreted as quantum interference in phase space [36]. The parity signal is directly visible in the histograms shown in Fig. 4 for different evolution times and most pronounced at short times, where spurious single-spin rotation terms proportional to $\hat{S}_{i}^{z}$ are smallest and atom number decay is negligible. We characterized the evolution of the parity of the number of detected atoms in $|\rightarrow\rangle$ by evaluating $\langle\hat{P}\rangle=\left\langle e^{-i \pi \sum_{i=1}^{N} \hat{S}_{i}}\right\rangle$, with $\hat{S}_{i}^{\rightarrow}=|\rightarrow\rangle_{i}\left\langle\left.\rightarrow\right|_{i}\right.$, detecting if atom $i$ is in the $|\rightarrow\rangle$ state. The parity is a sensitive measure of the many-body coherence in a sense that it detects decoherence-induced loss, spin flips or dephasing of every spin in the chain [23]. For the initial state at $t=0$, we obtain $\langle\hat{P}\rangle=0.41(7)$, where the reduction of the parity compared to unity is expected because of imperfections in the microwave rotations and decoherence attributable to magnetic field noise, both leading to uncorrelated rotations of individual spins, even in the absence of Rydberg dressing [23]. Subsequently, the observed parity signal decays with increasing dressing time $t$, and we extract a time constant of $\tau_{P}=0.13(4) \mathrm{ms}$, which is on the order of $\tau / N$, the characteristic time to lose a single atom. Hence, we conclude that off-resonant excitation to the Rydberg state, followed by a loss of the excited atom, is the dominating decoherence effect [23]. 


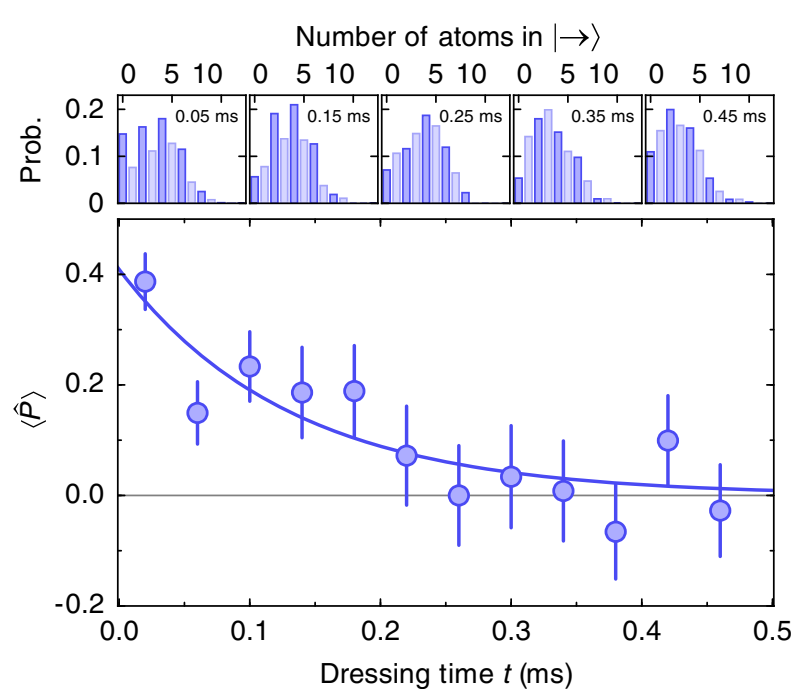

FIG. 4. Evolution of the spin parity in the initially unpopulated $|\rightarrow\rangle$ state. The histograms on top show the evolution of the number of detected atoms in the $|\rightarrow\rangle$ state with even numbers highlighted by the darker color. The data were binned in intervals of $0.1 \mathrm{~ms}$ and the bin centers are indicated. The main plot shows the extracted parity $\langle\hat{P}\rangle$ vs time, together with an exponential fit with time constant $\tau_{P}=0.13(4) \mathrm{ms}$ (solid line). All error bars denote one s.e.m.

\section{CONCLUSION}

Our observations of coherent, interaction-driven collapse and revival dynamics establish Rydberg dressing as a promising technique to study interacting spin systems also in more complex scenarios [37,38]. Indeed, the demonstrated interaction to decay ratio of $2 \pi U_{0} \tau \approx 100$ exceeds our previous result [18] by a factor of 100 and is comparable to the state of the art for implementing spin Hamiltonians in ion chains [10,11,39]. The additional factor of $2 \pi$ has been included to follow the convention used in previous publications $[10,11,18]$. While in the current experiment faster dynamics at larger interaction strengths was not accessible because of merely technical limitations in the pulse timing accuracy, the interaction strength and hence the interaction-to-decay ratio can be significantly increased by working closer to resonance at a larger Rydberg state admixture. The improved available long coherence times combined with the controlled, optically induced interactions allow for the implementation of Loschmidt-echo-type sequences to characterize the value of the generated states for quantum metrological applications [26]. The study of periodically driven systems in two dimensions or in systems with periodic boundary conditions are now also within reach, holding promise to shed new light on many-body phases existing solely in nonequilibrium scenarios $[40,41]$, among them the "Floquet time crystal" phase $[39,42,43]$. Furthermore, our experiments mark the first step towards a Rydberg quantum annealer based on coherent Rydberg-dressed interactions
[44] and the study of itinerant quantum matter with longrange, soft-core interactions $[22,45,46]$.

\section{ACKNOWLEDGMENTS}

We thank Simon Hollerith for proofreading the manuscript and Benoit Vermersch for discussions. We acknowledge support by the Danish National Research Foundation (DNRF) through a Niels Bohr Professorship for T.P. as well as funding by Max-Planck-Gesellschaft (MPG); European Union (EU) (Ultracold Quantum Matter, Rydberg Quantum Simulators, Marie Curie Fellowship to J. C.); Deutsche Forschungsgemeinschaft (DFG) through the SPP 1929 (GiRyd); and the Körber Foundation.

[1] S. Lloyd, Universal Quantum Simulators, Science 273, 1073 (1996).

[2] W. Lechner, P. Hauke, and P. Zoller, A Quantum Annealing Architecture with All-to-All Connectivity from Local Interactions, Sci. Adv. 1, e1500838 (2015).

[3] G. Rempe, H. Walther, and N. Klein, Observation of Quantum Collapse and Revival in a One-Atom Maser, Phys. Rev. Lett. 58, 353 (1987).

[4] M. Brune, F. Schmidt-Kaler, A. Maali, J. Dreyer, E. Hagley, J. M. Raimond, and S. Haroche, Quantum Rabi Oscillation: A Direct Test of Field Quantization in a Cavity, Phys. Rev. Lett. 76, 1800 (1996).

[5] D. M. Meekhof, C. Monroe, B. E. King, W. M. Itano, and D. J. Wineland, Generation of Nonclassical Motional States of a Trapped Atom, Phys. Rev. Lett. 76, 1796 (1996).

[6] G. Kirchmair, B. Vlastakis, Z. Leghtas, S. E. Nigg, H. Paik, E. Ginossar, M. Mirrahimi, L. Frunzio, S. M. Girvin, and R. J. Schoelkopf, Observation of Quantum State Collapse and Revival Due to the Single-Photon Kerr Effect, Nature (London) 495, 205 (2013).

[7] M. Greiner, O. Mandel, T. W. Hänsch, and I. Bloch, Collapse and Revival of the Matter Wave Field of a Bose-Einstein Condensate, Nature (London) 419, 51 (2002).

[8] S. Will, T. Best, U. Schneider, L. Hackermüller, D.-S. Lühmann, and I. Bloch, Time-Resolved Observation of Coherent Multi-Body Interactions in Quantum Phase Revivals, Nature (London) 465, 197 (2010).

[9] R. Blatt and C. F. Roos, Quantum Simulations with Trapped Ions, Nat. Phys. 8, 277 (2012).

[10] P. Jurcevic, B. P. Lanyon, P. Hauke, C. Hempel, P. Zoller, R. Blatt, and C.F. Roos, Quasiparticle Engineering and Entanglement Propagation in a Quantum Many-Body System, Nature (London) 511, 202 (2014).

[11] P. Richerme, Z.-X. Gong, A. Lee, C. Senko, J. Smith, M. Foss-Feig, S. Michalakis, A. V. Gorshkov, and C. Monroe, Non-Local Propagation of Correlations in Quantum Systems with Long-Range Interactions, Nature (London) 511, 198 (2014).

[12] J. G. Bohnet, B. C. Sawyer, J. W. Britton, M. L. Wall, A. M. Rey, M. Foss-Feig, and J. J. Bollinger, Quantum Spin Dynamics and Entanglement Generation with Hundreds of Trapped Ions, Science 352, 1297 (2016). 
[13] B. Yan, S. A. Moses, B. Gadway, J. P. Covey, K. R. A. Hazzard, A. M. Rey, D. S. Jin, and J. Ye, Observation of Dipolar Spin-Exchange Interactions with Lattice-Confined Polar Molecules, Nature (London) 501, 521 (2013).

[14] A. de Paz, A. Sharma, A. Chotia, E. Maréchal, J. H. Huckans, P. Pedri, L. Santos, O. Gorceix, L. Vernac, and B. Laburthe-Tolra, Nonequilibrium Quantum Magnetism in a Dipolar Lattice Gas, Phys. Rev. Lett. 111, 185305 (2013).

[15] P. Schauß, J. Zeiher, T. Fukuhara, S. Hild, M. Cheneau, T. Macrì, T. Pohl, I. Bloch, and C. Gross, Crystallization in Ising Quantum Magnets, Science 347, 1455 (2015).

[16] H. Labuhn, D. Barredo, S. Ravets, S. de Léséleuc, T. Macrì, T. Lahaye, and A. Browaeys, Tunable Two-Dimensional Arrays of Single Rydberg Atoms for Realizing Quantum Ising Models, Nature (London) 534, 667 (2016).

[17] Y.-Y. Jau, A. M. Hankin, T. Keating, I. H. Deutsch, and G. W. Biedermann, Entangling Atomic Spins with a RydbergDressed Spin-Flip Blockade, Nat. Phys. 12, 71 (2016).

[18] J. Zeiher, R. van Bijnen, P. Schauß, S. Hild, J.-y. Choi, T. Pohl, I. Bloch, and C. Gross, Many-Body Interferometry of a Rydberg-Dressed Spin Lattice, Nat. Phys. 12, 1095 (2016).

[19] D. Barredo, H. Labuhn, S. Ravets, T. Lahaye, A. Browaeys, and C. S. Adams, Coherent Excitation Transfer in a Spin Chain of Three Rydberg Atoms, Phys. Rev. Lett. 114, 113002 (2015).

[20] L. Santos, G. V. Shlyapnikov, P. Zoller, and M. Lewenstein, Bose-Einstein Condensation in Trapped Dipolar Gases, Phys. Rev. Lett. 85, 1791 (2000).

[21] I. Bouchoule and K. Mølmer, Spin Squeezing of Atoms by the Dipole Interaction in Virtually Excited Rydberg States, Phys. Rev. A 65, 041803 (2002).

[22] N. Henkel, R. Nath, and T. Pohl, Three-Dimensional Roton Excitations and Supersolid Formation in Rydberg-Excited Bose-Einstein Condensates, Phys. Rev. Lett. 104, 195302 (2010).

[23] See Supplemental Material at http://link.aps.org/ supplemental/10.1103/PhysRevX.7.041063 for more details.

[24] J. M. Radcliffe, Some Properties of Coherent Spin States, J. Phys. A: Gen. Phys. 4, 313 (1971).

[25] K. R. A. Hazzard, M. van den Worm, M. Foss-Feig, S. R. Manmana, E. G. Dalla Torre, T. Pfau, M. Kastner, and A. M. Rey, Quantum Correlations and Entanglement in Far-from-Equilibrium Spin Systems, Phys. Rev. A 90, 063622 (2014).

[26] T. Macrì, A. Smerzi, and L. Pezzè, Loschmidt Echo for Quantum Metrology, Phys. Rev. A 94, 010102 (2016).

[27] M. Foss-Feig, Z.-X. Gong, A. V. Gorshkov, and C. W. Clark, Entanglement and Spin-Squeezing without InfiniteRange Interactions, arXiv:1612.07805.

[28] R. Raussendorf and H. J. Briegel, A One-Way Quantum Computer, Phys. Rev. Lett. 86, 5188 (2001).

[29] M. Foss-Feig, K. R. A. Hazzard, J. J. Bollinger, and A. M. Rey, Nonequilibrium Dynamics of Arbitrary-Range Ising Models with Decoherence: An Exact Analytic Solution, Phys. Rev. A 87, 042101 (2013).

[30] J. Schachenmayer, B. P. Lanyon, C. F. Roos, and A. J. Daley, Entanglement Growth in Quench Dynamics with Variable Range Interactions, Phys. Rev. X 3, 031015 (2013).
[31] C. Weitenberg, M. Endres, J. F. Sherson, M. Cheneau, P. Schauß, T. Fukuhara, I. Bloch, and S. Kuhr, Single-Spin Addressing in an Atomic Mott Insulator, Nature (London) 471, 319 (2011).

[32] T. Fukuhara, A. Kantian, M. Endres, M. Cheneau, P. Schauß, S. Hild, D. Bellem, U. Schollwöck, T. Giamarchi, C. Gross, I. Bloch, and S. Kuhr, Quantum Dynamics of a Mobile Spin Impurity, Nat. Phys. 9, 235 (2013).

[33] T. Fukuhara, S. Hild, J. Zeiher, P. Schauß, I. Bloch, M. Endres, and C. Gross, Spatially Resolved Detection of a Spin-Entanglement Wave in a Bose-Hubbard Chain, Phys. Rev. Lett. 115, 035302 (2015).

[34] M. Cheneau, P. Barmettler, D. Poletti, M. Endres, P. Schauß, T. Fukuhara, C. Gross, I. Bloch, C. Kollath, and S. Kuhr, Light-Cone-Like Spreading of Correlations in a Quantum Many-Body System, Nature (London) 481, 484 (2012).

[35] G. Breitenbach, S. Schiller, and J. Mlynek, Measurement of the Quantum States of Squeezed Light, Nature (London) 387, 471 (1997).

[36] W. Schleich and J.A. Wheeler, Oscillations in Photon Distribution of Squeezed States and Interference in Phase Space, Nature (London) 326, 574 (1987).

[37] A. W. Glaetzle, M. Dalmonte, R. Nath, C. Gross, I. Bloch, and P. Zoller, Designing Frustrated Quantum Magnets with Laser-Dressed Rydberg Atoms, Phys. Rev. Lett. 114, 173002 (2015).

[38] R. M. W. van Bijnen and T. Pohl, Quantum Magnetism and Topological Ordering via Rydberg Dressing near Förster Resonances, Phys. Rev. Lett. 114, 243002 (2015).

[39] J. Zhang, P. W. Hess, A. Kyprianidis, P. Becker, A. Lee, J. Smith, G. Pagano, I.-D. Potirniche, A. C. Potter, A. Vishwanath, N. Y. Yao, and C. Monroe, Observation of a Discrete Time Crystal, Nature (London) 543, 217 (2017).

[40] V. Khemani, A. Lazarides, R. Moessner, and S. L. Sondhi, Phase Structure of Driven Quantum Systems, Phys. Rev. Lett. 116, 250401 (2016).

[41] I.-D Potirniche, A. C. Potter, M. Schleier-Smith, A. Vishwanath, and N. Y. Yao, Floquet Symmetry-Protected Topological Phases in Cold-Atom Systems, Phys. Rev. Lett. 119, 123601 (2017).

[42] D. V. Else, B. Bauer, and C. Nayak, Floquet Time Crystals, Phys. Rev. Lett. 117, 090402 (2016).

[43] S. Choi, J. Choi, R. Landig, G. Kucsko, H. Zhou, J. Isoya, F. Jelezko, S. Onoda, H. Sumiya, V. Khemani, C. von Keyserlingk, N. Y. Yao, E. Demler, and M. D. Lukin, Observation of Discrete Time-Crystalline Order in a Disordered Dipolar Many-Body System, Nature (London) 543, 221 (2017).

[44] A. W. Glaetzle, R. M. W. van Bijnen, P. Zoller, and W. Lechner, A Coherent Quantum Annealer with Rydberg Atoms, Nat. Commun. 8, 15813 (2017).

[45] M. Mattioli, M. Dalmonte, W. Lechner, and G. Pupillo, Cluster Luttinger Liquids of Rydberg-Dressed Atoms in Optical Lattices, Phys. Rev. Lett. 111, 165302 (2013).

[46] A. Geißler, I. Vasić, and W. Hofstetter, Condensation vs Long-Range Interaction: Competing Quantum Phases in Bosonic Optical Lattice Systems at Near-Resonant Rydberg Dressing, Phys. Rev. A 95, 063608 (2017). 